\title{
Research of Factors Affecting Orientation of PAN Precursor used in Carbon Fiber
}

\author{
Yue Tang ${ }^{a}$, Fubin Hou ${ }^{b}$ \\ School of Qingdao University of Science and Technology, Qingdao 266061, China \\ akedaty@126.com, bfirstmanme@qq.com
}

Keywords: PAN, degree of orientation, dry-wet spinning.

\begin{abstract}
In the process of producing PAN precursor used in carbon fiber, applying orthogonal experiment method and a dry-wet spinning technology, the main systematical study are the influence of coagulation bath, jet stretch, pre-draft and water-bath draft on degree of orientation. Results show that under applying dry-wet spinning technology, in smaller jet velocity, and to give fiber appropriate pre-draft ratio and high water-bath draft ratio, degree of orientation is higher, and high fiber strength also can be obtained.
\end{abstract}

\section{Introduction}

Carbon fiber is a kind of fiber material with more than $90 \%$ of carbon content, possessing the high strength, high specific modulus, conductive and heat resistant and self-lubricating excellent comprehensive performance, applied in aviation, aerospace, automotive, electronics, machinery, chemical industry, sports equipment and other fields widely. After oxidation carbonization and surface treatment of PAN precursor, the carbon fiber based on PAN is produced one of the most widely used high performance fiber materials and is also currently the fastest growing ${ }^{[1]}$.

The quality of the carbon fiber to a large extent depends on the quality of PAN, and the orientation degree of PAN is the main factors influencing the mechanical properties of PAN ${ }^{[2-3]}$. In the process of the carbon fiber based on PAN production, it is necessary to improve the quality of carbon fiber to explore the influence factors of the original silk orientation degree and to improve the quality of PAN. This paper adopts a certain molecular weight PAN spinning solution and the application of dry-Jet wet spinning, as well as explore the influence of the coagulation bath, shower and the pre-tension and hot water draft on orientation degree of the original silk, at last speculating the best conditions of orientation degree of the original silk.

\section{Experimentation}

Spinning solution and the spinning forming. With DMSO as solvent and AIBN as initiator, choose AN acrylic acid solution to polymerize to get good composite spinning solution ${ }^{[4]}$.

Slurry filter after metering pumps measurement, and then jet through nozzle. Apply dry-Jet wet spinning. The whole process is as follows: Homogeneous solution of polymerization, polymerization, filter, reaeration, metrology, dry-wet spinning, pre-draft, wash, water-bath stretch, heat-setting, silk.

Test. Coagulation bath concentration can be got through measuring refractive index under $20^{\circ} \mathrm{C}$ by Abbe refractometer.

Fineness of fibers can be determined by conventional weighing method of fixed-length.

Strength of fibers can be measured by YG021-A, a type of single wire electronic tonometer. Measurement conditions: length $10 \mathrm{~mm}$, speed $10 \mathrm{~mm} / \mathrm{min}$.

Sonic orientation can use Som-ii type sonic orientation Analyzer. Measurement conditions: length $40 \mathrm{~cm}$, tension about $0.1 \mathrm{cN} / \mathrm{dtex}$. 


\section{Outcome and discussion}

Determine the forming process parameters. Orthogonal design method can be utilized to determine the parameters. By selecting jet speed, jet draft ratio, pre-draft ratio and water-bath draft velocity as four factors, orthogonal experiment of four factors and three levels will be available as shown in Tab. 1.

Tab.1 Element level table

\begin{tabular}{ccccc}
\hline & $\begin{array}{c}\text { jet speed } \\
(\mathrm{m} / \mathrm{min})\end{array}$ & $\begin{array}{c}\text { jet draft ratio } \\
\text { (times) }\end{array}$ & $\begin{array}{c}\text { pre-draft ratio } \\
\text { (times) }\end{array}$ & $\begin{array}{c}\text { water-bath draft velocity } \\
\text { (m/min) }\end{array}$ \\
\hline 1 & 14.12 & 0.8 & 1.2 & 40.06 \\
\hline 2 & 20.04 & 1 & 1.5 & 60.12 \\
\hline 3 & 25.18 & 1.2 & 1.8 & 80.03 \\
\hline
\end{tabular}

Orthogonal test result and analysis is shown in Tab.2.

By Visual analysis on T value from test results, the best level of jet rate, Jet stretch ratio, pre-draft ratio and water-bath draft rate is respectively 1,3,3,3. Seeing from results of range $\mathrm{R}$, these factors of effects on degree of orientation rank according to the strength: water-bath stretch rate $>$ jet speed $>$ jet stretch radio $>$ pre-stretch radio. With improve of and water-bath rate, degree of orientation rises, when jet speed increases, degree of orientation declines. Effect of pre-draft on degree of orientation is compared complex, existing a minimum value. Above shows that to obtain a high degree of orientation, it is necessary to high jet draft radio, water-bath stretch rate, lower jet speed, as well as proper Pre-stretch radio. Besides when degree of orientation grow, the fiber strength also rise, which indicate this analysis is reasonable.

Tab.2 Orthogonal test result and analysis

\begin{tabular}{|c|c|c|c|c|c|c|}
\hline & $\begin{array}{c}\text { jet } \\
\text { velocity }\end{array}$ & $\begin{array}{l}\text { jet draft } \\
\text { ratio }\end{array}$ & $\begin{array}{c}\text { pre-draf } \\
\mathrm{t} \\
\text { ratio } \\
\end{array}$ & $\begin{array}{l}\text { water-bath draft } \\
\text { velocity }\end{array}$ & $\begin{array}{c}\text { fiber } \\
\text { strength } \\
\text { (cN/dtex) }\end{array}$ & $\begin{array}{c}\text { Degree of } \\
\text { orientation (\%) }\end{array}$ \\
\hline 1 & 1 & 1 & 3 & 2 & 3.65 & 61.25 \\
\hline 2 & 1 & 2 & 2 & 1 & 2.92 & 55.05 \\
\hline 3 & 1 & 3 & 1 & 3 & 4.96 & 68.37 \\
\hline 4 & 2 & 1 & 1 & 1 & 2.86 & 49.10 \\
\hline 5 & 2 & 2 & 3 & 3 & 3.78 & 64.64 \\
\hline 6 & 2 & 3 & 2 & 2 & 3.26 & 58.45 \\
\hline 7 & 3 & 1 & 2 & 3 & 3.14 & 55.85 \\
\hline 8 & 3 & 2 & 1 & 2 & 3.27 & 57.62 \\
\hline 9 & 3 & 3 & 3 & 1 & 3.02 & 52.01 \\
\hline $\mathrm{T} 1$ & 184.67 & 166.20 & 175.09 & 156.16 & & \\
\hline $\mathrm{T} 2$ & 172.19 & 177.31 & 169.35 & 177.32 & & \\
\hline T3 & 165.48 & 178.83 & 177.90 & 188.86 & & \\
\hline R1 & 12.48 & -11.11 & 5.74 & -24.16 & & \\
\hline R2 & 6.71 & -1.52 & -8.55 & -11.54 & & \\
\hline
\end{tabular}

Temperature and concentration of coagulating bath. In this experiment, addition to temperature and concentration of coagulating bath, other terms refer to the orthogonality condition $5 \#$ to set. Firstly by changing Temperature rating of coagulation bath the influence of temperature on degree of orientation can be investigated. The results are presented in Tab.3.

Tab.3 influence of temperature of coagulation bath on degree of orientation

\begin{tabular}{cccc}
\hline temperature rating & 1 & 2 & 3 \\
\hline fiber strength (cN/dtex) & 4.16 & 3.42 & 3.21 \\
\hline Degree of orientation (\%) & 77.18 & 72.13 & 69.78 \\
\hline
\end{tabular}

Tab. 3 shows that with rise of temperature of the coagulation bath, degree of orientation decrease, meanwhile fiber strength decline. This is because during process of solidification solvent with coagulant can generate a double diffusion effect ${ }^{[5]}$. When the temperature rises, the diffusion 
coefficient increases, and then double diffusion process is accelerated, with fiber forming speeding and average pore radius and average fiber radius soaring. These lead to uneven and severe defects in internal structure, and therefore degree of orientation and fiber strength decrease.

Next only change concentration rating of coagulation bath to explore and other terms refer to the orthogonality $5 \#$ to set. Results presented in Tab.4 show that in a certain range, increase of concentration of coagulation bath accompany rise of degree of orientation of fiber, but when the concentration is increased to a certain value, the influence will weaken. This is because as the concentration increase, filament silk get slowed or draft ratio of filament in coagulating bath lower, leading to diminution of diffusion coefficient of solvent or coagulant, with forming of double diffusion process eased, so fiber structures tend to be dense and evenly.

Tab.4 influence of concentration of coagulation bath on degree of orientation

\begin{tabular}{cccc}
\hline Concentration rating & 1 & 2 & 3 \\
\hline Fiber strength(cN/dtex) & 3.24 & 3.96 & 3.91 \\
\hline Degree of orientation (\%) & 58.12 & 64.35 & 63.92 \\
\hline
\end{tabular}

Pre-draft and water-bath draft. Thermal stabilization of tension stretch is the prerequisite for producing high performance carbon fiber ${ }^{[6]}$.After setting that the overall draft ratio is 3.8 and jet stretch ratio is 1.5,and remaining the stretch ratio, as well as other conditions, the effect of pre-draft and water-bath draft on degree of orientation as shown in Tab 5. When degree of orientation falls,

Tab.5 the effect of pre-draft and water-bath draft on degree of orientation,

\begin{tabular}{cccc}
\hline Pre-draft ratio & Water-bath draft ratio & Fiber strength(cN/dtex) & Degree of orientation (\%) \\
\hline 1.4 & 2.71 & 5.39 & 69.44 \\
\hline 1.5 & 2.53 & 3.95 & 63.82 \\
\hline 2.0 & 1.90 & 2.57 & 51.25 \\
\hline
\end{tabular}

Water-bath draft ratio drops down significantly, indicating water-bath draft have great effects on deree of orientation; After setting a certain pre-draft ratio, in order to improve degree of orientation and increase strength, water-bath draw ratio should be given maximum.

Dry heat draft. When proceeding hot drawing, firstly the fibers are put in dry heat air. Then under specific temperature and other conditions, the effect of stretching ratio on degree of orientation is detected, as shown in Tab.6. When the draw ratio increases, degree of orientation will rise, and strength also will increase. But after stretching ratio more than 1.7, degree of orientation will began to decline, the strength also will follow down, at the same time there appear. This is because when the draft ratio is too large, although the degree of orientation increase, due to that the stretching speed quickens, tensile time will be shortened under the high temperature, so it is difficult to relax he internal stress which comes from drawing, leading to the existence of internal stress that will decrease the strength of the fiber, and generate easily caused by local rift tow, which is not conducive to dry heat drawing ${ }^{[7]}$.

Tab.6 the impact of draft ratio on degree of orientation

\begin{tabular}{ccccccccc}
\hline Draft ratio & 1.2 & 1.3 & 1.4 & 1.45 & 1.6 & 1.7 & 1.77 & undrawn \\
\hline Fiber strength(cN/dtex) & 4.35 & 5.08 & 5.20 & 5.67 & 6.07 & 6.53 & 6.26 & 3.90 \\
\hline Degree of orientation (\%) & 73.50 & 76.40 & 76.95 & 78.35 & 80.04 & 84.36 & 83.24 & 66.65 \\
\hline remark & & & & & & \multicolumn{3}{c}{ fuzziness } \\
\hline
\end{tabular}

After setting certain draw ratio, by changing the air temperature, there examine the influence of drawing temperature on the degree of orientation ,as shown Tab.7 ,indicating that under the same stretching ratio, with the improvement of drawing temperature, degree of fiber orientation and strength increase. This is because there are CN groups containing strong polarity on the PAN macromolecular chain, besides between $\mathrm{CN}$ groups exist a great deal of repulsive force and gravity because of their difference of position inside and outside, which adverse to activity of the macromolecular chain, and then causes twists and turns in its local ${ }^{[7]}$. Its strong polarity about $\mathrm{CN}$ groups also has great attraction between the molecules and forms many join points easily, granting the fiber larger rigidity which can reduce or disappear only at higher temperatures. Therefore, the strength of fiber showed dependence on the temperature in the process of heat draft. 
Tab.7 the influence of temperature in the process of dry heat draft

\begin{tabular}{cccc}
\hline Temperature grade & 1 & 2 & 3 \\
\hline Fiber strength(cN/dtex) & 5.62 & 6.07 & 6.35 \\
\hline Degree of orientation (\%) & 78.26 & 80.12 & 83.26 \\
\hline
\end{tabular}

Water-bath draft. With the dry heat draft conditions, position the fiber in water bath, heat stretching is proceeded. By changing temperatures or stretch ratio, results of measured degree of orientation are shown in Tab.8, indicating that with the increase of temperature and stretch ratio, degree of orientation also increase.

Tab.8 the impact of temperature and draft ratio on degree of orientation in water-bath draft

\begin{tabular}{cccc}
\hline Temperature & 1 & 2 & 3 \\
\hline Draft ratio & 1.8 & 2.0 & 2.5 \\
\hline Degree of orientation (\%) & 88.2 & 89.6 & 91.9 \\
\hline
\end{tabular}

\section{Summary}

Under the condition of not influence on jet, jet speed should be as small as possible, and the relatively higher the jet draft ratio is, the better.

The temperature of coagulation bath should be appropriately reduced, and the concentrations should be appropriate elevated.

The pre-draft shouldn't be too large.

The fiber is suitable for dry and water-bath drafting, draft ratio can be relative the higher and the better, but also is unfavorable for excess, otherwise leading to easily appearance of fuzziness which affect the quality of PAN.

\section{References}

[1] Yue Zhang, Yingwu Chen. Research Development of PAN — based Carbon Fibers. Fiber composites , Vol. 1 (2009) No.1, p.7-10.

[2] Shujuan Yu, Lijun,Jiang. Progress of Preparation Technology of PAN Precursor Used in Carbon Fiber,Hi-tech Fiber \&Application,Vol.28(2003) No.6, p.15-18.

[3] Chenguo Wang, Bo Zhu. ANALYSIS OF RESTRICTING THE DEVELOPMENT OF CARBON FIBER IN OUR COUNTRY. JOURNAL OF SHANDONG UNIVERSITY(ENGINEERING SCIENCE. Vol. 32(2002) No.6, p. 521-525.

[4] Xiaofeng Jiang, Ruqing Ni. PREPARING FOR HIGH PERFORMANCE PAN PRECURSOR. Synthetic Fiber in China. Vol.29 (2000) No.4, p. 23-26.

[5] Xiaoping Cai, Production Technology of Polyacrylonitrile based Carbon Fiber, Chemical Industry Press, China, 2012.

[6] Pinghua Wang, Jie Liu. THE CHANGE OF FIBER TENSION DURING THE CONTINUOUS PRE-OXIDATION OF PAN PRECURSOR FIBERS. China Synthetic Fiber Industry, Vol.14 (1991) No.5, p. 32-35.

[7] Guangzhu Li. Polymer Materials Processing Technology, China Textile Press. China, 2010, p. 136-137. 